\title{
The Mesenchymal-Like Phenotype of the MDA-MB-231 Cell Line
}

\author{
Khoo Boon Yin \\ Institute for Research in Molecular Medicine (INFORMM), \\ Universiti Sains Malaysia, Penang, \\ Malaysia
}

\section{Introduction}

Mesenchymal stem cells (MSCs) are progenitor cells that can be isolated from all connective tissues such as bone, adipose, cartilage, blood and muscle (Wang et al., 2009). MSCs have recently been described to localise within breast carcinomas where the stem cells integrate into tumour-associated stromal tissues whereby the MSCs promote breast cancer cell invasion and metastasis (Karnoub et al., 2007). Previous studies have demonstrated that when combine with weakly metastatic human breast carcinoma cells, bone marrow-derived mesenchymal stem cells (BMSCs) increase the metastatic potency of the cancer cells greatly (Hombauer \& Minguell, 2000). This phenomenon was significantly observed in MCF-7 cells where increase in cancer cell proliferation was observed when the cancer cells were co-cultured on the BMSCs feeder layer. Furthermore, light and epifluorescence microscopy studies revealed that the MCF-7 cluster grew in a dispersed fashion on the BMSCs feeder layer due to the decrease expression of adhesive molecules, such as E-cadherin and epithelial-specific antigen (ESA), in the cancer cells. The interaction between the MCF-7 cells and the BMSCs likely causes the loss of the adhesive molecules in the cancer cells. A phenomenon similar to this interaction was also observed in our recent study. Indeed, the study found that the growth of the MCF-7 cells was enhanced not only when the cancer cells were adhesively co-cultured with the BMSCs but also when they were co-cultured non-adhesively.

In the adhesive cell interaction, the growth or proliferation rate of the MCF-7 cells, which was measured by colony size, was observed to increase when the cancer cells were cocultured on the BMSCs feeder layer (Fig. 1A and Fig. 1B). The non-adhesive interaction of the MCF-7 cells with BMSCs was also found to increase the growth of the cancer cells. When the cancer cells were incubated with the conditioned medium (culture supernatant) of the BMSCs, the proliferation rate of the MCF-7 cells increased approximately $16.6 \%$ when compared to the proliferation rate of the cancer cells incubated with growth medium only (Fig. 1C). This phenomenon indicates that the increase in the proliferation rate of the cancer cells due to the presence of the BMSCs must not be related to a direct physical cell-cell interaction, as similar findings are observed in both the adhesive and non-adhesive coculture conditions.

Note: In this chapter, adhesive co-culture is defined as the growth of cancer cells on a nontumorigenic cell monolayer where direct physical cell-cell interaction occurs. Non-adhesive 
co-culture is defined as the incubation of cancer cells with a conditioned medium that is withdrawn from the non-tumorigenic cells; here, the cells interact with one another via the culture medium.

(A)

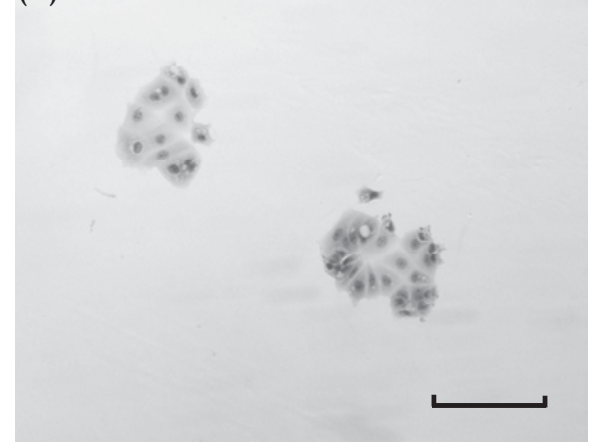

(C)
(B)

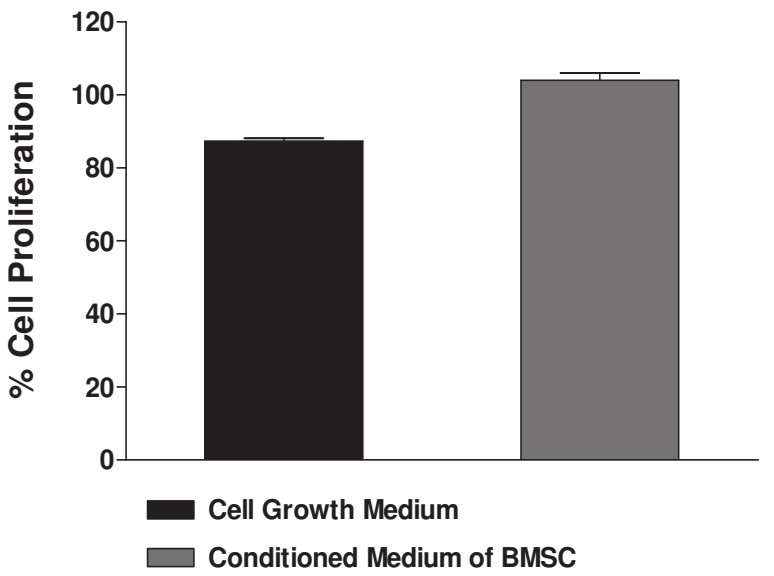

Fig. 1. Colony formation of the MCF-7 cells cultured with or without BMSCs. The pictures show Oil-Red-O staining of (A) the MCF-7 colonies alone and (B) the MCF-7 colonies grown on the BMSCs feeder layer for one week. The pictures were visualised under an inverted light microscope using same magnification. (C) The proliferation rate of the MCF-7 cells incubated with cell growth medium (control) and conditioned medium of BMSCs for one week. One hundred cells were used as the input prior to incubation. The values were expressed as mean $\pm \mathrm{SD}$ from three replicates, and the determination was carried out from three replicates each of three independent experiments.

The BMSCs likely secreted or influenced the MCF-7 cells to secrete certain soluble growth factors into the conditioned medium whereby the growth factors stimulated the MCF-7 cells in cluster and grow into single cell layer, after which the cells dispersed without any 
evidence of direct cell-cell contact (Fig. 2). Thus, the influence of the BMSCs on the changed of cell morphology and increased proliferation rate of the MCF-7 cells may be achieved via the culture medium without the need for any direct physical cell-cell interaction between the two cell lines. However, this phenomenon, in which the BMSCs increased the proliferation rate of the breast cancer cells, was not observed when the BMSCs were cocultured with highly invasive and metastatic human breast cancer cells, such as MDA-MB231 cells. The MDA-MB-231 cell line likely contains its own source that similar to the MSCs as progenitor factor in the cell population that is able to secrete a standard level of the soluble growth factors into the conditioned medium of the MDA-MB-231 cells. Therefore, the activity of the MDA-MB-231 cells was not influenced by their exposure to the MSCsconditioned medium and few effects were observed when the cells were co-cultured with the MSCs (Sasser et al., 2007a).
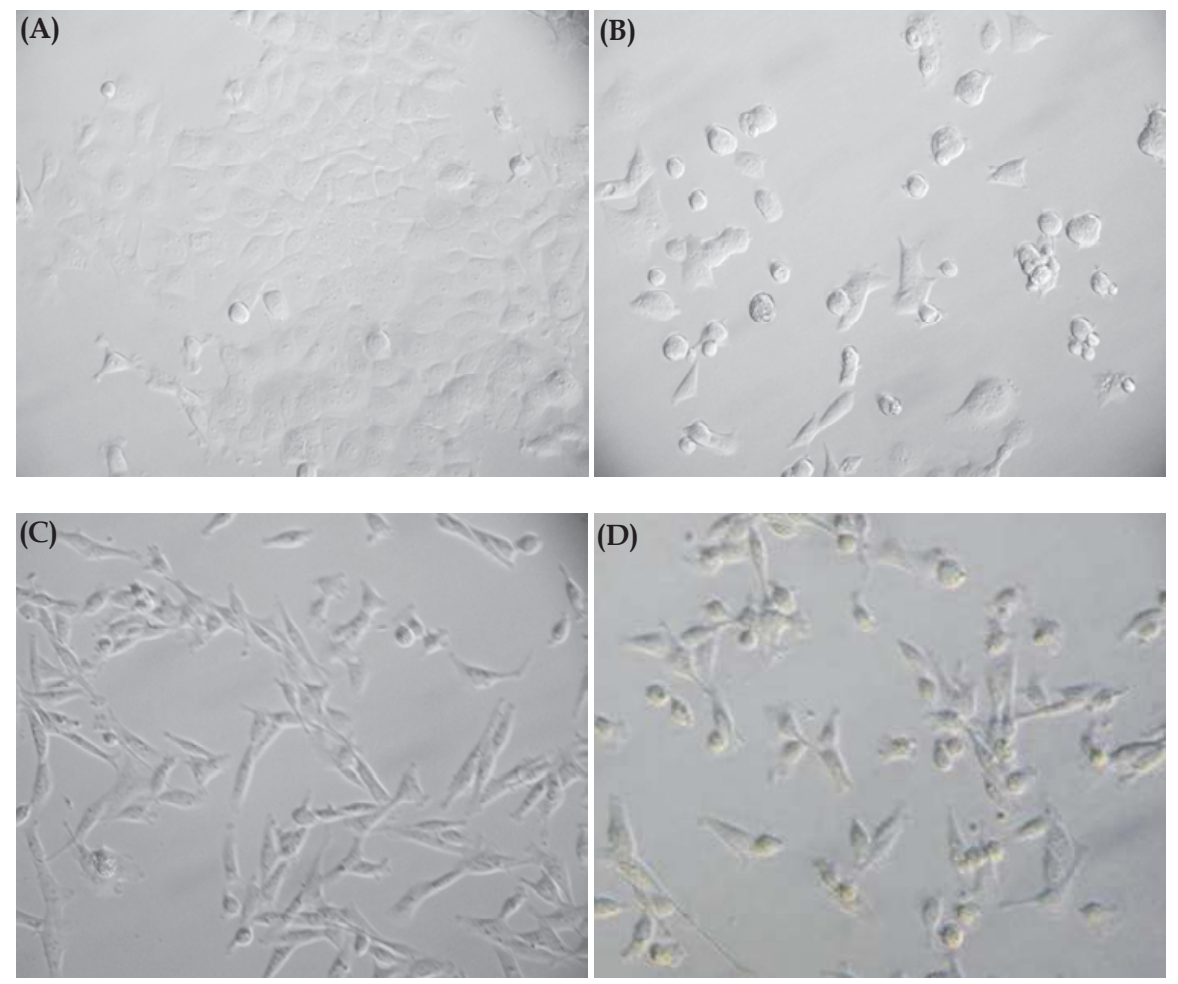

Fig. 2. Unstained MCF-7 and MDA-MB-231 cells as visualised under an inverted light microscope. Panels A and B show clustered and single cell layer of MCF-7 cells that were incubated with cell growth medium and BMSCs-conditioned medium, respectively, whereas panels $\mathbf{C}$ and $\mathbf{D}$ show few effects on cell morphology were observed when the MDA-MB-231 cells were incubated with cell growth medium and BMSCs-conditioned medium, respectively. The pictures were taken after one week of cell incubations. 


\section{Soluble growth factors in the conditioned medium of the MDA-MB-231 cells}

\subsection{Expression of MMPs in the conditioned medium}

The MDA-MB-231 cell line is an estrogen receptor alpha (ER $\alpha)$-negative human breast cancer cell line (Liu et al., 2003). It was derived from a metastatic adenocarcinoma of the mammary gland of a 51-year-old Caucasian woman, according to the data sheet of the American Type Culture Collection (ATCC). This adherent epithelial cell line that likely contains more than one cell populations is a highly aggressive, invasive and poorlydifferentiated human breast cancer cell line. Similar to other invasive cancer cell lines, the MDA-MB-231 cells display the invasiveness by mediating the proteolytic degradation of the extracellular matrix (ECM), including basement membrane and several mechanical barriers to the ECM, through the increased expression of matrix metalloproteinases (MMPs), including gelatinases, en route to their destinations (Fig. 3).

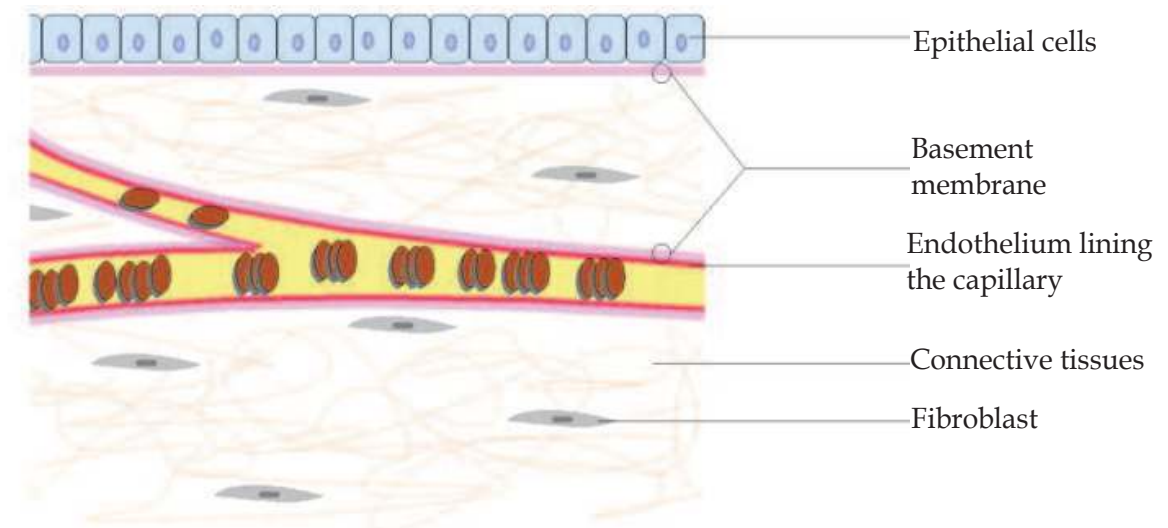

Fig. 3. Illustration depicts the ECM in relationship to the epithelium, endothelium and connective tissues. To reach their destination, the invasive cancer cells must penetrate the mechanical barriers of the ECM and basement membrane through proteolytic degradation. The figure was modified from the Wikimedia.

Type IV collagen, which is the main component of the basement membrane, is the first component that must be degraded to allow the invasion process (Boutaud et al., 2000). The ability to degrade and penetrate the basement membrane is related with an increased potential of the cells for invasion and metastasis (Castro-Sanchez et al., 2011). Tumour cells are able to produce MMPs that degrade the matrix barriers surrounding the tumour, including basement membrane, permitting invasion into connective tissues, entry and exit from blood and lymphatic vessels, and metastasis to distant organs. MMPs are family of zinc-dependent endopeptidases that collectively are capable of degrading all components of the ECM, including the basement membrane. Binding of breast cancer cells to type IV collagen in the basement membrane induces Discoidin domain receptor 1 (DDR1) activation and then it triggers signal transduction pathways and cellular processes that promotes secretion of MMPs which contributes to basement membrane degradation and cancer cell 
invasion. A previous study demonstrated that gelatinase B or MMP-9, which degrades the type IV collagen in the basement membrane, plays a crucial role in the invasion process of the MDA-MB-231 cells (Liu et al., 2003). This phenomenon can be observed by determining the metastatic potential of the MDA-MB-231 cells in an experimental model that is closely correlated with the expression of the MMP-9 and the activities of the gelatinases in the conditioned medium of the MDA-MB-231 cells. According to the study, the invasion of the MDA-MB-231 cells was blocked by MMP-9-neutralising antibodies that reduced the gelatinolytic activities in the conditioned medium, as detected using Enzyme-linked immunosorbent assay (ELISA). This phenomenon also led to the significant inhibition of the invasive capacities of the MDA-MB-231 cells. This inhibition was induced by specific drugs e.g., peroxisome proliferator-activated receptor gamma ligands and all-trans-retinoic acid that were administered on a reconstituted basement membrane in a Matrigel ${ }^{\circledR}$ chamber in vitro. Therefore, MMP-9 was shown to play a crucial role in the invasion process of the MDA-MB-231 cells and it was shown to be absolutely required for the transmigration of this cell line.

Note: In this chapter, conditioned medium is denoted as culture supernatant that is withdrawn from feeder layer. To accomplish this, a culture of feeder layer e.g., BMSCs is maintained with fresh growth medium. After certain duration, the growth medium is withdrawn from the feeder layer as conditioned medium. The conditioned medium is believed to contain growth factors released by the feeder layer.

\subsection{Activation of STAT3 and soluble IL-6 in the conditioned medium}

In addition to MMP-9 in the conditioned medium, the MDA-MB-231 cells are also demonstrated to contain elevated level of signal transducer and activator of transcription 3 (STAT3) in the cells (Sasser et al., 2007b). STAT3 is typically maintained in the cytoplasm as an inactive monomer. Once it is phosphorylated, the STAT3 forms homodimers and enters into nucleus where it activates the transcription of multiple genes associated with cell proliferation and survival (Heinrich et al., 1998; Zinzalla et al., 2010). The activation of STAT3 has been correlated with enhanced breast cancer cell growth, survival and immune evasion (Selander et al., 2004; Ling et al., 2005; Yu et al., 2007). According to a previous study, exposure of MSCs-conditioned medium to MCF-7 and T-47D activated the levels of pTyr ${ }^{705}$ STAT3 in the cells (Sasser et al., 2007a). Correlatively, the enhancement of the cancer cell growth rates was observed in ER $\alpha$-positive human breast cancer cell lines, including MCF-7 and T-47D, in the presence of the MSCs-conditioned medium. The growth rates of BT474 and ZR-75-1 cells were also observed to increase after the cancer cells were co-cultured with the MSCs-conditioned medium. All cancer cell growth rates were enhanced by approximately 2-3 fold, after the exposure to the conditioned medium (Fig. 4). The growth rate of an ER $\alpha$-negative breast cancer cell line, MDA-MB-468, was also elevated in the presence of the MSCs-conditioned medium, albeit to a lesser extent than the other ER $\alpha$ positive cell lines that were tested (Sasser et al., 2007a; Sasser et al., 2007b). However, this induction was not observed when the MDA-MB-231 cell line was exposed to the MSCsconditioned medium.

Few effects were observed when the MDA-MB-231 cells were co-cultured with the MSCsconditioned medium because the cell line likely contained a subpopulation in the cell population that secreted a standard level of soluble growth factors in the conditioned medium (Sasser et al., 2007b). In this non-adhesive co-culture study, paracrine interleukin-6 


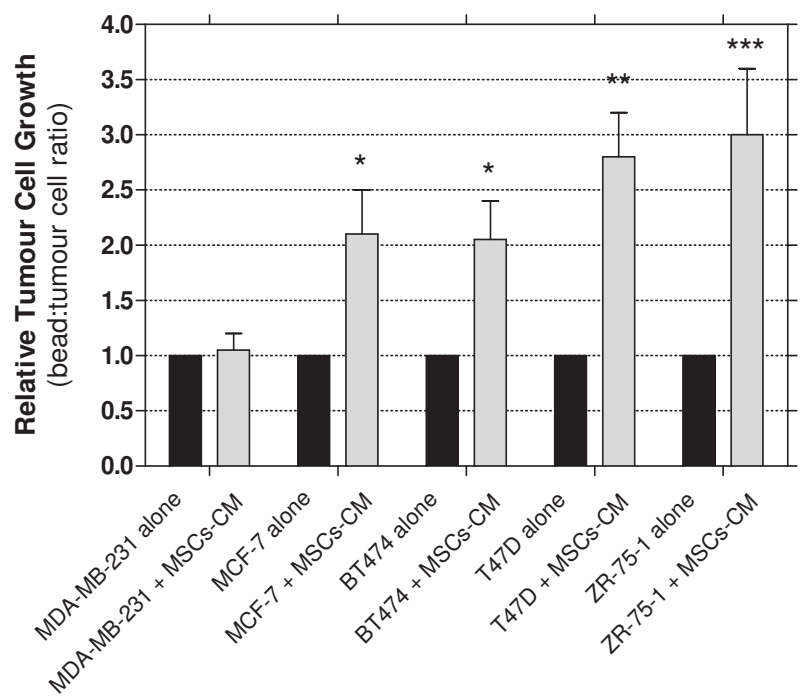

Fig. 4. Breast cancer cell growth in the presence or absence of MSCs-conditioned medium was assessed for MDA-MB-231, MCF-7, BT474, T47D and ZR-75-1 cells. The MDA-MB-231 cell growth was unaltered by MSCs-conditioned medium, whereas the growth of the four remaining ER $\alpha$-positive cell lines in the presence of MSCs-conditioned medium was significantly elevated when compared to the cell lines growing alone for eight days (Sasser et al., 2007b).

(IL-6) was found to be the principal mediator of the STAT3 phosphorylation in the cells. MSCs-induced STAT3 phosphorylation was lost when the IL-6 was depleted from the MSCs-conditioned medium. A similar phenomenon was observed when the IL-6 receptor in the cancer cells was blocked. This secretion of IL-6 from the MDA-MB-231 cells allowed for the activation and maintenance of the level of STAT3 as well as the growth in the MDA-MB231 cells. Therefore, the conditioned medium of MDA-MB-231 cells has similar effect as the conditioned medium withdrawal from the MSCs, as evidenced by previous study, where the conditioned medium from the MDA-MB-231 cells with constitutively active STAT3 is sufficient to induce p-STAT3 levels in various recipients that do not possess elevated pSTAT3 levels, such as MCF-10A cells, a non-tumorigenic cell line (Lieblein et al., 2008). This signalling occurs through the JAK/STAT3 pathway, leading to STAT3 phosphorylation as early as 30 minutes and was persistent for at least 24 hours, indicating that a correlation between elevated levels of IL-6 production and p-STAT3 in the cells, as confirmed by ELISA analysis. Neutralisation of the IL-6 ligand or gp130 was sufficient to block the increased levels of p-STAT3 (Y705) in the treated cells. These results demonstrate that the STAT3 phosphorylation in breast epithelial cells can be stimulated by paracrine signalling through soluble growth factors from both breast cancer cells and breast cancer associated fibroblasts with elevated STAT3 phosphorylation. The finding of growth factors within the MDA-MB231 conditioned media was also sufficient to stimulate an increase in IL-6 production from 
MCF-10A cells, as indicated in the previous study, may not correct as both MDA-MB-231 cells and MCF-10A cells secret IL-6 in the conditioned medium. Indeed, our study demonstrated that the conditioned media of the MDA-MB-231 cells and MCF-10A cells contained a high level of IL-6, although the level was not as high as in the MSCs-conditioned medium (Fig. 5).

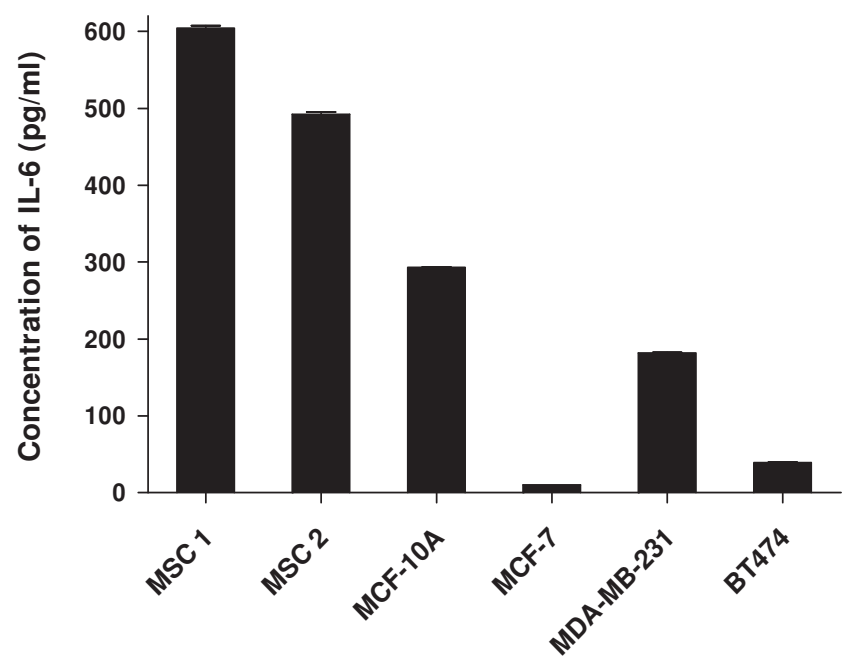

Fig. 5. The activity of IL-6 in the conditioned media of MSCs, non-tumorigenic cells and human breast cancer cells. The culture supernatants were withdrawn from one-week-old feeder layer of above cultures. The level of IL-6 in the conditioned medium was assayed using ELISA. The values were expressed as the mean \pm SD from three replicates, and the determination was carried out from three replicates each of three independent experiments.

Therefore, the finding indicates that the soluble growth factors within the MDA-MB-231 conditioned medium to stimulate an increase in IL-6 production from the MCF-10A cells might be due to combine of both conditioned media. Anyhow, this result indicates that the MDA-MB-231 cells may contain similar progenitor factor as MSCs in the cell population. This factor likely expresses the high level of IL-6 where it contributes to the induction of STAT3 phosphorylation and appears to be associated with cell proliferation of the MDAMB-231 cells. Although the secretion of IL- 6 allows for the activation and maintenance of the level of STAT3 as well as the growth in the MDA-MB-231 cells have been demonstrated, its role in invasiveness of the MDA-MB-231 remains unclear. Nevertheless, targeting the IL-6 in the conditioned medium can be an idea to diagnose patients with tumour that are ER $\alpha$ negative or express lower level of ER $\alpha$.

\subsection{CCL2 and CCL5 in the conditioned medium}

Chemokines or chemotactic cytokines are small proteins that are classified into four conserved groups, CXC, CC, C and CX3C, based on the position of the first two cysteines that are adjacent to the amino acid (Balkwill, 2004; Lu \& Kang, 2009). Among more than 50 
identified human chemokines, chemokine (C-C motif) ligand 2 (CCL2 or MCP-1) and chemokine (C-C motif) ligand 5 (CCL5 or RANTES) are of particularly important. CCL2 is a potent chemoattractant for monocytes, memory $\mathrm{T}$ lymphocytes and natural killer cells whereas CCL5 is a potent inducer of leukocyte motility (Lu \& Kang, 2009; Melgarejo et al., 2009; YaalHahoshen et al., 2006). Both chemokines stimulate migration of leukocytes in response to inflammatory signals. The roles of CCL2 and CCL5 in breast malignancy have been extensively addressed in breast cancer studies (Goldberg-Bittman et al., 2004; Soria et al., 2008; Soria \& Ben-Baruch, 2008; Wu et al., 2008; Fujimoto et al., 2009). Overexpression of the CCL2 and CCL5 are stimulated during breast cancer development and progression. They are also frequently associated with advanced tumour stage and metastatic relapse in breast cancer. Both chemokines act directly on the tumour cells to promote their pro-malignancy phenotype by increasing their migratory and invasion-related properties (Soria \& Ben-Baruch, 2008). The chemokines are expressed by the cells of the tumour microenvironment osteoblasts and MSCs. In breast cells, the chemokines are highly expressed by breast tumour cells at primary tumour sites and minimally expressed by normal breast epithelial duct cells (Soria et al., 2008; Soria \& Ben-Baruch, 2008). The chemokines are soluble growth factors that can be easily detected in serum and conditioned culture medium. Consistently, our recent study demonstrated that high levels of CCL2 and CCL5 were detected in the conditioned medium of the MDA-MB-231 cells, as determined by ELISA (Fig. 6). The results indicated that the CCL2 and CCL5 were present in the conditioned media of all of the tested cell lines. As expected, elevated levels of CCL2 and CCL5 were observed in the MSCs and MCF-10A cells. CCL2 was additionally more stably expressed in the non-tumorigenic cells, such as MCF-10A, than in the MDA-MB-231 cells. However, an opposite event was observed for CCL5 in the MCF-10A and MDA-MB-231 cells. Both CCL2 and CCL5 displayed relatively higher expression levels in the MDA-MB-231 cells than that in the weakly metastatic cells, such as MCF-7 and BT-474. However, the CCL2 level in the MDA-MB-231 cells was only slightly higher than in the MCF-7 and BT-474 cells.

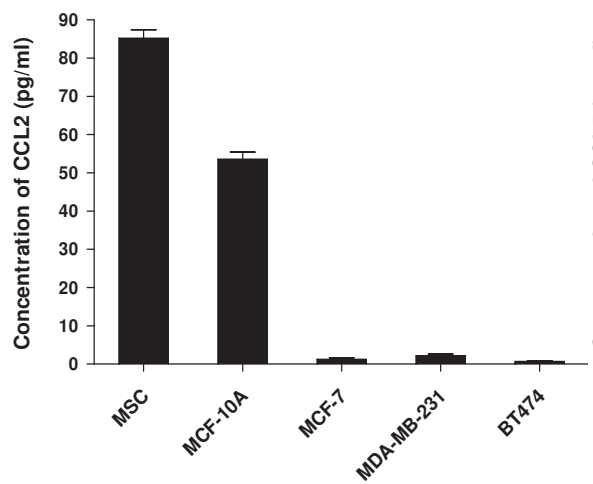

(A)

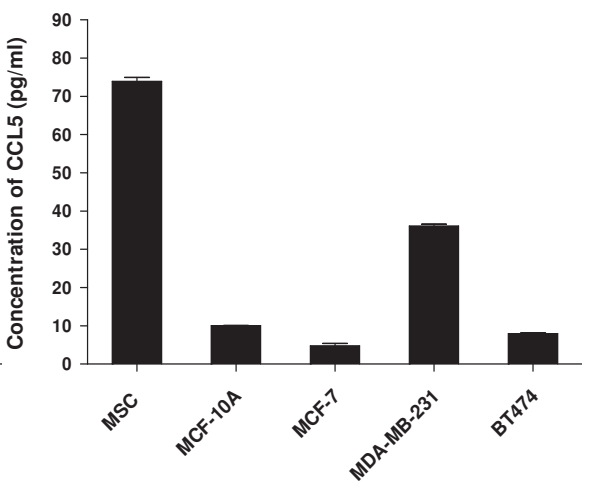

(B)

Fig. 6. The activities of (A) CCL2 and (B) CCL5 in the conditioned media of MSCs, human breast tumorigenic and non-tumorigenic cells. The levels of the soluble growth factors were assayed using ELISA. The values were expressed as mean \pm SD from three replicates, and the determination was carried out from three replicates each of three independent experiments. 
The overexpression of chemokine decoy receptor proteins, such as D6 and Duffy antigen receptor for chemokines (DARC), have been demonstrated to inhibit the proliferation and invasion of the human breast cancer in vitro, tumorigenesis and lung metastasis in vivo (Wu et al., 2008). This inhibition is associated with decrease in chemokines, such as CCL2 and CCL5, vessel density and tumour-associated macrophage infiltration. The inhibition of CCL5 expression by short interfering RNA (siRNA) or by the use of neutralising antibodies against CCL5 impaired the tumour-supporting roles that were mediated by the CCL5-CCR5 loop; this significantly inhibited the metastatic potential of the MDA-MB-231 cells (Karnoub et al., 2007; Soria \& Ben-Baruch, 2008). Moreover, CCL2-neutralizing antibodies inhibited bone resorption in vitro and bone metastasis in vivo as well as the tumour conditioned media-induced osteoclast formation in vitro and bone metastasis in vivo, indicating a role of the CCL2 and CCL5 in metastasis (Lu \& Kang, 2009). The MDA-MB-231 cells are obviously having its own progenitor factor, which is in the cell population that produce the soluble growth factors in order to maintain the invasive and progressive phenotypes in the cells. Further identification and functional characterisation of CCL2 and CCL5, as well as MMP-9 and IL-6, would provide an effective treatment for systemic metastasis. Perhaps, the effects of certain inhibitors or drugs on the inhibition of proliferation and the reduction of invasion of breast cancer cell growth can be easily determined using these growth factors as they can be detected via serum and conditioned culture medium. Thus, all four molecules mentioned in this chapter could be considered as potential therapeutic targets for the development of a detection assay for human breast cancer.

\section{The subpopulation in the MDA-MB-231 cells}

Most of the cancer cell lines have recently been demonstrated by flow cytometry to contain a subpopulation of CD44+/CD24- where the MDA-MB-231 cells are found to contain a high percentage of the $\mathrm{CD} 44^{+} / \mathrm{CD} 24$ - subpopulation $(85 \pm 5 \%)$ in the cells (Sheridan et al., 2006). Other cell lines that contain a high level of this subpopulation are MDA-MB-436 (72 $\pm 5 \%), ~ H s 578 T \quad(86 \pm 5 \%)$ and SUM1315 (97 $\pm 3 \%)$ (Table 1). The subpopulation is shown to possess the capacity for self-renewal and the generation of heterogeneous progeny in the cells. Moreover, the subpopulation of the breast cancer cells has been reported to have stem/progenitor cell properties that contribute a unique ability to allow these cells to invade. Similar to the ability of the MSCs that was described above, the inherent properties of this subpopulation may impart their transformed counterparts with the ability to evade traditional antitumour therapies and to establish breast cancer metastasis (Reya et al., 2001; Behbod et al., 2005; Dean et al., 2005). Several studies suggested that this subpopulation of cells, as a subset of human breast cancer cells, possessed an enhanced ability to form tumour in immunocompromised mice (Al-Hajj et al., 2003; Ponti et al., 2005). However, the potential of this subpopulation to establish breast cancer metastasis in the cell line remains unclear.

The expression levels of pro-invasive genes, such as interleukin-1-alpha (IL-1 $\alpha$ ), IL-6, interleukin-8 (IL-8) and urokinase plasminogen activator (UPA), are higher in the cell lines that contained a significant CD44+ ${ }^{+}$CD24- subpopulation (Sheridan et al., 2006). The results indicate that the cell lines with a significant number of CD44 $/$ CD24- subpopulation are more invasive is consistent with the studies that demonstrate the metastatic process in breast cancer cells requires the following: (1) ECM degradation-associated proteins, including the 
UPA/UPA receptor system and MMPs; (2) cytokines, including interleukin-1 (IL-1), IL-6, IL-8, interleukin-11 (IL-11), tumour necrosis factor (TNF) and transforming growth factor-beta 1 (TGF- $\beta 1$ ); and (3) chemokines and their receptors, including stromal cell-derived factor-1alpha (SDF-1 $\alpha$ ) and CXC chemokine receptor (CXCR4) (Edwards and Murphy, 1998; Dumont and Arteaga, 2003; Kang et al., 2003; Yodkeeree et al., 2010). In addition, a recent study described the role of nuclear factor-kappa B (NF-кB) and its ligand in the metastasis of breast cancer cells to the bone matrix (Jones et al., 2006). All of these factors may be directly related to breast cancer metastasis. However, the contribution of the subpopulation of CD44+/CD24- to the pro-invasive factors in breast cancer cells remains unclear.

\begin{tabular}{cccc}
\hline No. & Cell Line & CD44 $^{+}$CD24- $^{-}$ & Cell Type Classification \\
\hline $\mathrm{a}$ & MDA-MB-231 & $85 \pm 5$ & Mesenchymal \\
\hline $\mathrm{b}$ & MDA-MB-436 & $72 \pm 5$ & Myoepithelial \\
\hline $\mathrm{c}$ & Hs578T & $86 \pm 5$ & Mesenchymal \\
\hline $\mathrm{d}$ & MDA-MB-468 & $3 \pm 1$ & Basal \\
\hline $\mathrm{e}$ & MCF-7 & 0 & Luminal \\
\hline $\mathrm{f}$ & T47-D & 0 & Luminal \\
\hline $\mathrm{g}$ & ZR-75-1 & 0 & Luminal \\
\hline $\mathrm{h}$ & BT-474 & 0 & Luminal/ErbB2 ${ }^{+}$ \\
\hline $\mathrm{i}$ & SK-BR-3 & 0 & Basal \\
\hline $\mathrm{j}$ & MCF-10A & $17 \pm 4$ & \\
\hline
\end{tabular}

Table 1. Subpopulation of CD44+/CD24- in commonly used breast cancer cell lines. The CD44 and CD24 expression patterns in the subpopulation CD44 ${ }^{+} / \mathrm{CD} 24-$ were determined by flow cytometry. CD44 and CD24 were detected by a combination of fluorochromeconjugated monoclonal antibodies against human CD44 (FITC) and CD24 (PE), respectively (Sheridan et al., 2006).

Demethoxycurcumin (DMC) is recently demonstrated to inhibit the adhesion, migration and invasion of the MDA-MB-231 cells (Yodkeeree et al., 2010). According to the study, the DMC-treated MDA-MB-231 cells contained decreasing levels of ECM degradationassociated proteins, which included MMP-9, membrane type-1 MMP (MT1-MMP), UPA and UPAR. DMC reduced also the expression of intercellular adhesion molecule-1 (ICAM-1) and CXCR4 in the MDA-MB-231 cells. These molecules are involved in the modulation of the tumour metastasis process. In addition, the study showed that treatment of the MDA-MB231 cells with DMC inhibited the DNA binding activity of NF- $\kappa \mathrm{B}$, which is known to mediate the expression of MMPs, UPA, UPAR, ICAM-1 and CXCR4 in breast cancer cells. These results indicated also that NF- $\mathrm{KB}$ may play a role in the invasion process of the MDAMB-231 cells. All of these findings suggest the presence of a correlation between the above molecules and the invasiveness of the MDA-MB-231 cells. However, the specific correlation between the above molecules and the subpopulation in the MDA-MB-231 cells has yet to be fully elucidated. The inhibition or depletion of the progenitor factor from the subpopulation is hypothesised to reduce the expression of the above molecules, thereby reducing the invasiveness of the MDA-MB-231 cells. Therefore, it is essential to identify more surface markers that can specifically be used to isolate the subpopulation. By targeting this subpopulation in the cells, the expression of the above molecules and the invasiveness of the MDA-MB-231 cells can be further elucidated. 


\section{Mesenchymal-like phenotype of the MDA-MB-231 cells}

Before the subpopulation and invasiveness of the MDA-MB-231 cells can be further elucidated, hierarchy of the breast cancer stem cells in the breast cancer cell compartment should be understood. A breast cancer stem cell, as described in the cancer stem cell compartment hierarchy, is capable of undergoing an asymmetric cell division to generate one cell that is identical to itself (orange colour) and one that it is more committed towards a certain differentiation pattern (a breast cancer cell, grey colour) (Cariati \& Purushotham, 2008; Fig. 7).

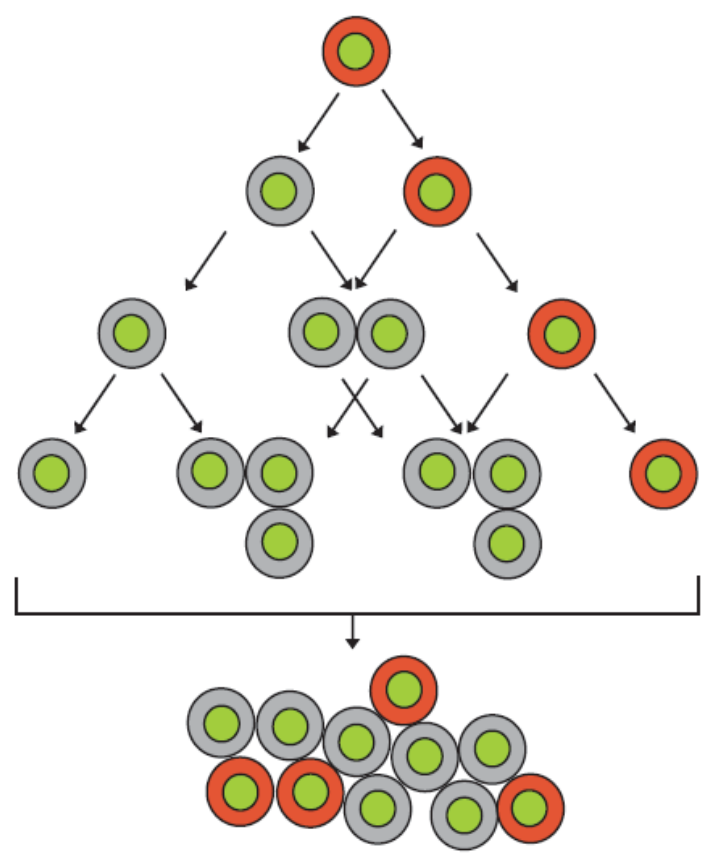

Fig. 7. A breast cancer stem cells-breast cancer cells compartment hierarchy. A breast cancer stem cell is capable of going through an asymmetric cell division to generate one cell that is identical to itself (orange colour) and one that tends toward a certain differentiation pattern (breast cancer cells, grey colour) (Modified from Cariati \& Rurushotham, 2008).

The formation of the identical cell ensures that the cancer stem cell compartment is maintained throughout its time in the subpopulation. These distinct cells undergo series of divisions and differentiation steps that result in the generation of a terminally differentiated 
population of breast cancer stem cells and breast cancer cells. The existence of these cancer stem cells explains why only a small minority of cancer cells are capable of extensive proliferate and transfer to the tumour. Chemotherapy can remove breast cancer cells, but it fails to eliminate the cancer stem cells that can revive the breast cancer cells. This allows the regrowth of the breast cancer after the treatment has ended (Sanchez-Garcia et al., 2008). This shortcoming explains the high recurrence of the disease. Therefore, the current strategy for the development of anti-breast cancer agents is to target both breast cancer stem cells and breast cancer cells. Moreover, the healthy breast cells transform into cancer cells via the formation of breast cancer stem cells is also a possibility, but, the precise mechanism of the transformation for this disease remains unclear. Therefore, study of the transformation remains warranted. By understanding the precise mechanism that transforms normal stem cells into cancer cells via the formation of the cancer stem cells, it would be possible to develop more effective tools for the ER $\alpha$-negative human breast cancer prevention, detection and treatment.

The MDA-MB-231 cell line is a good example of a breast cancer cell line that consists of the above mentioned populations of breast cancer stem cells and breast cancer cells. As described above, the MDA-MB-231 cells have been demonstrated to contain a subpopulation of $\mathrm{CD} 44^{+} / \mathrm{CD} 24-$ that provides stem/progenitor cell properties to enhance the invasiveness of the cancer cells. Surprisingly, in our present study, we found that the MDA-MB-231 cells were positive for CD105 staining, while the weakly metastatic breast cancer cell lines, such as MCF-7 and T47D, were negative for the CD105 staining; the staining was visualised by fluorescent microscopy (Fig. 8A). CD105, also known as Endoglin, is a type I integral trans-membrane glycoprotein and is an accessory receptor for transforming growth factor-alpha (TGF $\beta$ ) superfamily ligands (Barbara et al., 1999). CD105 is found on activated monocytes, mesenchymal stromal cells and leukemic cells of lymphoid and myeloid lineages. The BMSCs, as well as other non-hematopoietic MSCs, are positive for the CD105 antibody staining, as visualised by fluorescent microscopy (Miao et al., 2006; Bernacki et al., 2008). Therefore, it is hypothesised that the MDA-MB-231 cells may have similar mesenchymal phenotypes as the progenitor factors that contribute to the metastatic potency of the cancer cells. Therefore, the cell line contains not only the stem/progenitor cell properties but also the mesenchymal-like stem/progenitor cell properties. This property likely contributes to the invasiveness of the cell line and causes the cell line to express high levels of MMP-9, IL-6, CCL2 and CCL5 in the conditioned medium. The Oil-Red-O-stained MDA-MB-231 cells were also observed to contain a mixture of epithelial cells and a mesenchymal-like subpopulation, as visualised by light microscopy (Fig. 8B).

These results focused our attention and research on the mesenchymal-like phenotype in the MDA-MB-231 cells. MDA-MB-231 and MCF-7 are cell lines that originate from pleural effusion metastatic cells in ductal invasive breast carcinomas (Burdall et al., 2003; Lacroix \& Leclercq, 2004). These cells are among the most commonly used breast cancer cell lines in medical research laboratories. MDA-MB-231 is a mesenchymal-like cell line that is highly aggressive and invasive, whereas MCF-7 is classified as a luminal (epithelial)-like cell line with a relatively low invasive phenotype and potential (Lacroix \& Leclercq, 2004; Charafe-Jauffret et al., 2006). A comparison of the two cell lines in terms of DNA copy number variation and gene expression profiles has been performed, and the expression levels of 2157 transcripts were shown to be significantly increased in the MDAMB-231 
cells compared with the MCF-7 cells; the expression levels of 2345 transcripts were significantly increased in the MCF-7 cells compared with the MDA-MB-231 cells (Forozon et al., 2000; Charafe-Jauffret et al., 2006). Moreover, 387 of the above transcripts have been defined by the gene expression profile to be mesenchymal-like cellular subtypes (CharafeJauffret et al., 2006). Recently, 31 mesenchymal-like and luminal-like subtype features of breast cancer cell lines were revealed in Charafe-Jauffret's study, which was based on the gene expression profiles. The study found that 680 transcripts were preferentially expressed in the group of mesenchymal-like cell lines, and 629 transcripts were expressed preferentially in the group of luminal-like breast cancer cell lines. In a recent expression study, 387 transcripts, which are also identified in the mesenchymal-like subtype gene list in Charafe-Jauffret's study, showed significantly higher expression levels in the MDAMB-231 cells; and 328 transcripts, which were present on the luminal subtype gene list from Charafe-Jauffret's study, showed significantly higher expression levels in the MCF-7 cells ( $\mathrm{Li}$ et al., 2009). These data revealed the differential expression profiles of mesenchymal-like and luminal-like subtypes of the breast cancer cell lines. These expression profiles can be utilised to effectively overcome the invasion and metastasis of human breast cancer.
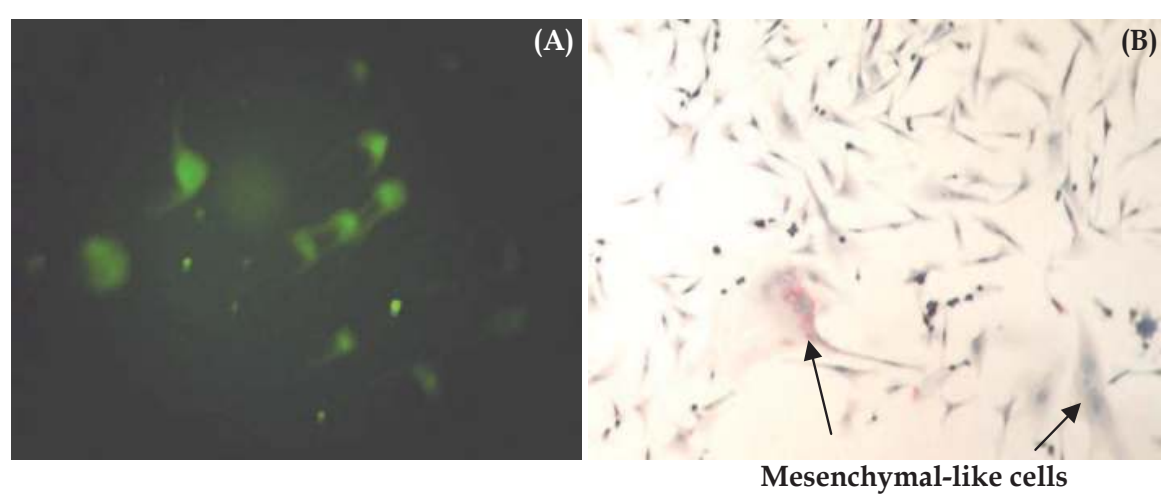

Fig. 8. A: The MDA-MB-231 cells, which were predicted to have a mesenchymal-like phenotype subpopulation, existed in the epithelial cell population. The cells were stained with Oil-red $\mathrm{O}$ and visualised using an inverted light microscope. B: The MDA-MB-231 cells were positive for the CD105 antibody staining as visualised using fluorescent microscope. These images were captured using a digital camera.

\section{Future prospects}

Our recent study proposes to isolate or withdraw the mesenchymal-like stem cells from the MDA-MB-231 population using CD105 and other known antibody-conjugated microbeads, thus allowing for a clearer understanding of the subpopulation of the cancer cells. Potential drugs will then be applied to the isolated CD105+ (mesenchymal-like stem 
cells) and CD105- (epithelial cells) MDA-MB-231 cells. The invasion rate of the drugtreated $\mathrm{CD}_{105^{+}}$and $\mathrm{CD}_{105}{ }^{-} \mathrm{MDA}-\mathrm{MB}-231$ will then be determined using the Matrigel invasion assay. The mRNA and protein expression levels of the ECM degradationassociated molecules in the drug-treated CD105+ and CD105- MDA-MB-231 cells will also be assessed using real-time PCR and Western Blotting, respectively, and the gelatinase activities in the conditioned medium of drug-treated CD105 ${ }^{+}$and CD105- MDA-MB-231 cells will be investigated using ELISA. The proposed project that will utilise cell separation and isolation techniques to study the breast cancer cell invasion is a new area of cancer research in the institute of my home country. The previous research projects regarding the MDA-MB-231 cell invasion and metastasis were related to drug treatments, the effects of herbal and plant extracts, and the understanding of a gene or protein activity in cancer cells and animal models. However, the approaches to study the cancer cell invasion and metastasis by isolating or withdrawing the mesenchymal-like breast cancer cells $\left(\mathrm{CD}_{\left.105^{+}\right)}\right.$from the MDA-MB-231 cell population using cell separation or isolation have not been demonstrated. Therefore, this project may establish a new fundamental cancer research and new research topic in my institute. This study may also lay a research foundation that is focused on the inhibition of invasion for the ER $\alpha$-negative human breast cancer cells. I also believe that, by targeting the mesenchymal-like phenotype in the MDA-MB-231 subpopulation, the invasion rate of the ER $\alpha$-negative human breast cancer cells can be easily monitored and controlled.

\section{Conclusion}

All of the results mentioned above show that the MDA-MB-231 cells likely display a mesenchymal-like phenotype that facilitates the cells to be a highly metastatic breast cancer cell line. However, a deeper understanding of the cell morphology, gene expression and intracellular mechanisms and pathways of the cancer cells that can explain the interaction between mesenchymal-like and epithelial cells in the MDA-MB-231 cells is warranted. By targeting this phenotype, the metastatic potency and the growth of the cancer cells may be controlled or effectively reduced. A potential anticancer drug can also be identified to treat both human breast cancers and other malignancies. Perhaps, withdrawing the progenitor factor from a tumour may serve as a potential machinery target in cell-mediated therapy for human breast cancer.

\section{Acknowledgements}

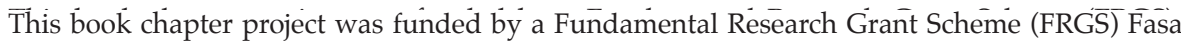
2/2010 (203/CIPPM/6711162) from the Ministry of Higher Education (MoHE), Malaysia and was partly supported by a Research University Grant Scheme for Individual (RUI) from Universiti Sains Malaysia (1001/CIPPM/811200).

\section{References}

Al-Hajj, M.; Wicha, M.S.; Benito-Hernandez, A.; Morrison, S.J. \& Clarke, M.F. (2003) Prospective identification of tumorigenic breast cáncer cells. Proc. Natl. Acad. Sci. USA, 100, 3983-3988. 
Balkwill, F. (2004) Cancer and the chemokine network. Nat. Rev. Cancer, 4,540-550.

Barbara, N.P.; Wrana, J.L. \& Letarte, M. (1999) Endoglin is an accessory protein that interacts with the signaling receptor complex of multiple members of the transforming growth factor-beta superfamily. J. Biol. Chem., 274, 584-594.

Behbod, F. \& Rosen, J.M. (2005) Will cancer stem cells provide new therapeutic targets? Carcinogenesis, 26, 703-711.

Bernacki, S.H.; Wall, M.E. \& Loboa, E.G. (2008) Isolation of human mesenchymal stem cells from bone and adipose tissue. Methods Cell Biol., 86, 257-278.

Boutaud, A.; Borza, D.B.; Bondar, O.; Gunwar, S.; Netzer, K.O.; Singh, N.; Ninomiya, Y.; Sado, Y.; Noelken, M.E. \& Hudson, B.G. (2000) Type IV collagen of the glomerular basement membrane. Evidence that the chain specificity of network assembly is encoded by the noncollagenous NC1 domains. J. Biol. Chem., 275, 30716-30724.

Burdall, S.E.; Hanby, A.M. \& Lansdown, M.R. (2003) Speirs V: Breast cancer cell lines: friend or foe? Breast Cancer Res., 5, 89-95.

Cariati, M. \& Purushotham, A.D. (2008) Stem cells and breast cancer. Histopathology, 52, 99_ 107.

Castro-Sanchez, L.; Soto-Guzman, A.; Guaderrama-Diaz, M.; Cortes-Reynosa, P.; Salazar, E.P. (2011) Role of DDR1 in the gelatinases secretion induced by native type IV collagen in MDA-MB-231 breast cancer cells. Clin. Exp. Metastasis, 28(5): 463-77.

Charafe-Jauffret, E.; Ginestier, C.; Monville, F.; Finetti, P.; Adelaide, J.; Cervera, N.; Fekairi, S.; Xerri, L.; Jacquemier, J.; Birnbaum, D. \& Bertucci, F. (2006) Gene expression profiling of breast cell lines identifies potential new basal markers. Oncogene, 25, 2273-2284.

Data sheet of ATCC for MDA-MB-231 cells, www.atcc.org/ATCCAdvancedCatalogSearch/ProductDetails/tabid/452/Default. aspx?ATCCNum=HTB-26\&Template=cellBiology

Dean, M.; Fojo, T. \& Bates, S. (2005) Tumour stem cells and drug resistance. Nat. Rev. Cancer, $5,275-284$.

Dumont, N. \& Arteaga, C.L. (2003) Targeting the TGF beta signaling network in human neoplasia. Cancer Cell, 3, 531-536.

Edwards, D.R. \& Murphy, G. (1998) Proteases: invasion and more. Nature, 394, 527-528.

Forozan, F.; Mahlamaki, E.H.; Monni, O.; Chen, Y.; Veldman, R.; Jiang, Y.; Gooden, G.C.; Ethier, S.P.; Kallioniemi, A. \& Kallioniemi, O.P. (2000) Comparative genomic hybridization analysis of 38 breast cancer cell lines: a basis for interpreting complementary DNA microarray data. Cancer Res., 60, 4519-4525.

Fujimoto, H.; Sangai, T.; Ishii, G.; Ikehara, A.; Nagashima, T.; Miyazaki, M. \& Ochiai, A. (2009) Stromal MCP-1 in mammary tumors induces tumour-associated macrophage infiltration and contributes to tumour progression. Int. J. Cancer, $125,1276-1284$.

Goldberg-Bittman, L.; Neumark, E.; Sagi-Assif, O.; Azenshtein, E.; Meshel, T.; Witz, I.P. \& Ben-Baruch, A. (2004) The expression of the chemokine receptor CXCR3 and its 
ligand, CXCL10, in human breast adenocarcinoma cell lines. Immunol Lett., 92, 171178.

Heinrich, P. C.; Behrmann, I.; Muller-Newen, G.; Schaper, F. \& Graeve, L. (1998) Interleukin6-type cytokine signaling through the gp130/Jak/STAT pathway. Biochem. J., 334, 297-314.

Hombauer, H. \& Minguell, J.J. (2000) Selective interactions between epithelial tumour cells and bone marrow mesenchymal stem cells. Br. J. Cancer, 82, 1290-1296.

Jones, D.H.; Nakashima, T.; Sanchez, O.H.; Kozieradzki, I.; Komarova, S.V.; Sarosi, I.; Morony, S.; Rubin, E.; Sarao, R.; Hojilla, C.V.; Komnenovic, V.; Kong, Y.Y.; Schreiber, M.; Dixon, S.J.; Sims, S.M.; Khokha, R.; Wada, T. \& Penninger, J.M. (2006) Regulation of cancer cell migration and bone metastasis by RANKL. Nature, 440, 692-696.

Kang, Y.; Siegel, P.M.; Shu, W.; Drobnjak, M.; Kakonen, S.M.; Cordon-Cardo, C.; Guise, T.A. \& Massague, J. (2003) A multigenic program mediating breast cancer metastasis to bone. Cancer Cell, 3, 537-549.

Karnoub, A.E.; Dash, A.B.; Vo, A.P.; Sullivan, A.; Brooks, M.W.; Bell, G.W.; Richardson, A.L.; Polyak, K.; Tubo, R. \& Weinberg, R.A. (2007) Mesenchymal stem cells within tumour stroma promote breast cancer metastasis. Nature, 449, 557-563.

Lacroix, M. \& Leclercq, G. (2004) Relevance of breast cancer cell lines as models for breast tumours: an update. Breast Cancer Res. Treat., 83, 249-289.

Li, J.; Gao, F.; Li, N.; Li, S.; Yin, G.; Tian, G.; Jia, S.; Wang, K.; Zhang, X.; Yang, H.; Nielsen, A.L. \& Bolund, L. (2009) An improved method for genome wide DNA methylation profiling correlated to transcription and genomic instability in two breast cancer cell lines. BMC Genomics. 10, 223.

Lieblein, J.C.; Ball, S.; Hutzen, B.; Sasser, A.K.; Lin, H.J.; Huang, T.H.; Hall, B.M. \& Lin, J. (2008) STAT3 can be activated through paracrine signaling in breast epithelial cells. BMC Cancer, 8, 302-315.

Ling, X. \& Arlinghaus, R. B. (2005) Knockdown of STAT3 expression by RNA interference inhibits the induction of breast tumors in immunocompetent mice. Cancer Res., 65, 2532-2536.

Liu, H.; Zang, C.; Fenner, M.H.; Possinger, K. \& Elstner, E. (2003) PPARgamma ligands and ATRA inhibit the invasion of human breast cancer cells in vitro. Breast Cancer Res. Treat., 79, 63-74.

Lu, X. \& Kang Y. (2009) Chemokine (C-C motif) ligand 2 engages CCR2+ stromal cells of monocytic origin to promote breast cancer metastasis to lung and bone. J. Biol. Chem., 284, 29087-29096.

Melgarejo, E.; Medina, M.A.; Sa'nchez-Jiménez, F.; \& Urdiales, J.L. (2009) Monocyte chemoattractant protein-1: a key mediator in inflammatory processes. Int. J. Biochem. Cell Biol., 41, 998-1001.

Miao, Z.; Jin, J.; Chen, L.; Zhu, J.; Huang, W.; Zhao, J.; Qian, H. \& Zhang, X. (2006) Isolation of mesenchymal stem cells from human placenta: comparison with human bone marrow mesenchymal stem cells. Cell Biol. Int., 30, 681-687.

Ponti, D.; Costa, A.; Zaffaroni, N.; Pratesi, G.; Petrangolini, G.; Coradini, D.; Pilotti, S.; Pierotti, M.A. \& Daidone, M.G. (2005) Isolation and in vitro propagation of 
tumorigenic breast cancer cells with stem/ progenitor cell properties. Cancer Res, 65, 5506-5511.

Reya, T.; Morrison, S.J.; Clarke, M.F. \& Weissman, I.L. (2001) Stem cells, cancer, and cancer stem cells. Nature, 414, 105-111.

Sanchez-Garcia, Isidro, Cobaleda and Cesar. 2008. Cancer Stem Cells. SciTopics. Retrieved May 20, 2010, from www.scitopics.com/Cancer_Stem_Cells.html.

Sasser, A.K.; Mundy, B.L.; Smith, K.M.; Studebaker, A.W.; Axel, A.E.; Haidet, A.M.; Fernandez, S.A. \& Hall, B.M. (2007a) Human bone marrow stromal cells enhance breast cancer cell growth rates in a cell line-dependent manner when evaluated in 3D tumour environments. Cancer Lett., 254, 255-264.

Sasser, A.K.; Sullivan, N.J.; Studebaker, A.W.; Hendey, L.F.; Axel, A.E. \& Hall, B.M. (2007b) Interleukin-6 is a potent growth factor for ER-alpha-positive human breast cancer. Faseb J., 21, 3763-3770.

Selander, K.S.; Li, L.; Watson, L.; Merrell, M.; Dahmen, H.; Heinrich, P.C.; Muller-Newen, G. \& Harris, K.W. (2004) Inhibition of gp130 signaling in breast cancer blocks constitutive activation of Stat3 and inhibits in vivo malignancy. Cancer Res., 64, 6924-6933.

Sheridan, C.; Kishimoto, H.; Fuchs, R.K.; Mehrotra, S.; Bhat-Nakshatri, P.; Turner, C.H.; Goulet, R.Jr.; Badve, S. \& Nakshatri, H. (2006) CD44+/CD24- breast cancer cells exhibit enhanced invasive properties: an early step necessary for metastasis. Breast Cancer Res., 8, R59.

Soria, G. \& Ben-Baruch, A. (2008) The inflammatory chemokines CCL2 and CCL5 in breast cancer. Cancer Lett., 267, 271-285.

Soria, G.; Yaal-Hahoshen, N.; Azenshtein, E.; Shina, S.; Leider-Trejo, L.; Ryvo, L.; Cohen-Hillel, E.; Shtabsky, A.; Ehrlich, M.; Meshel, T.; Keydar, I. \& Ben-Baruch, A. (2008) Concomitant expression of the chemokines RANTES and MCP-1 in human breast cancer: a basis for tumour-promoting interactions. Cytokine, 44, 191-200.

Wang, L.; Tran, I.; Seshareddy, K.; Weiss, M.L. \& Detamore, M.S. (2009) A comparison of human bone marrow-derived mesenchymal stem cells and human umbilical cordderived mesenchymal stromal cells for cartilage tissue engineering. Tissue Eng. Part A, 15, 2259-2266.

Wu, F.-Y.; Ou, Z.-L.; Feng, L.-Y.; Luo, J.-M.; Wang, L.-P.; Shen, Z.-Z. \& Shao, Z.-M. (2008). Chemokine decoy receptor D6 plays a negative role in human breast cancer. Molecular Cancer Research, 6, 1276-1288.

Yaal-Hahoshen, N.; Shina, S.; Leider-Trejo, L.; Barnea, I.; Shabtai, E.L.; Azenshtein, E.; Greenberg, I.; Keydar, I. \& Ben-Baruch, A. (2006) The Chemokine CCL5 as a Potential Prognostic Factor Predicting Disease Progression in Stage II Breast Cancer Patients. Clin. Cancer Res., 12, 4474-4480.

Yodkeeree, S.; Ampasavate, C.; Sung, B.; Aggarwal, B.B. \& Limtrakul, P. (2010) Demethoxycurcumin suppresses migration and invasion of MDA-MB-231 human breast cancer cell line. Eur. J. Pharmacol., 627, 8-15.

Yu, H.; Kortylewski, M. \& Pardoll, D. (2007) Crosstalk between cancer and immune cells: role of STAT3 in the tumour microenvironment. Nat. Rev. Immunol., 7, 41-51. 
Zinzalla, G.; Haque, M.R.; Basu, B.P.; Anderson, J.; Kaye, S.L.; Haider, S.; Hasan, F.; Antonow, D.; Essex, S.; Rahman, K.M.; Palmer, J.; Morgenstern, D.; Wilderspin, A.F.; Neidle, S. \& Thurston, D.E. (2010) A novel small-molecule inhibitor of IL-6 signalling. Bioorg. Med. Chem. Lett., 20, 7029-7032. 


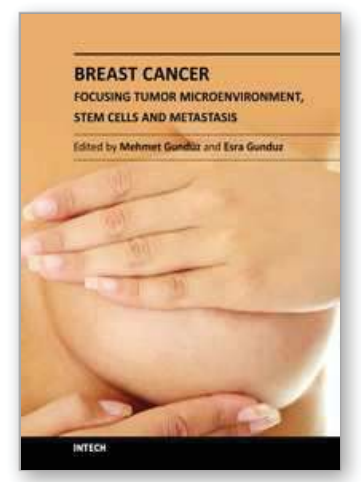

\author{
Breast Cancer - Focusing Tumor Microenvironment, Stem cells and \\ Metastasis \\ Edited by Prof. Mehmet Gunduz
}

ISBN 978-953-307-766-6

Hard cover, 584 pages

Publisher InTech

Published online 14, December, 2011

Published in print edition December, 2011

Cancer is the leading cause of death in most countries and its consequences result in huge economic, social and psychological burden. Breast cancer is the most frequently diagnosed cancer type and the leading cause of cancer death among females. In this book, we discussed characteristics of breast cancer cell, role of microenvironment, stem cells and metastasis for this deadly cancer. We hope that this book will contribute to the development of novel diagnostic as well as therapeutic approaches.

\title{
How to reference
}

In order to correctly reference this scholarly work, feel free to copy and paste the following:

Khoo Boon Yin (2011). The Mesenchymal-Like Phenotype of the MDA-MB-231 Cell Line, Breast Cancer Focusing Tumor Microenvironment, Stem cells and Metastasis, Prof. Mehmet Gunduz (Ed.), ISBN: 978-953307-766-6, InTech, Available from: http://www.intechopen.com/books/breast-cancer-focusing-tumormicroenvironment-stem-cells-and-metastasis/the-mesenchymal-like-phenotype-of-the-mda-mb-231-cell-line

\section{INTECH}

open science | open minds

\section{InTech Europe}

University Campus STeP Ri

Slavka Krautzeka 83/A

51000 Rijeka, Croatia

Phone: +385 (51) 770447

Fax: +385 (51) 686166

www.intechopen.com

\section{InTech China}

Unit 405, Office Block, Hotel Equatorial Shanghai

No.65, Yan An Road (West), Shanghai, 200040, China 中国上海市延安西路65号上海国际贵都大饭店办公楼 405 单元

Phone: +86-21-62489820

Fax: +86-21-62489821 
(C) 2011 The Author(s). Licensee IntechOpen. This is an open access article distributed under the terms of the Creative Commons Attribution 3.0 License, which permits unrestricted use, distribution, and reproduction in any medium, provided the original work is properly cited. 